\title{
Existence of zero-energy modes in the finite edged Kitaev honeycomb model
}

\author{
P Watts $\mathbf{S}^{1,2,3}$ and J Vala $\mathbf{a}^{1,2}$ \\ ${ }^{1}$ Department of Mathematical Physics, National University of Ireland, \\ Maynooth, Co. Kildare, Ireland \\ ${ }^{2}$ School of Theoretical Physics, Dublin Institute for Advanced Studies, \\ 10 Burlington Road, Dublin 4, Ireland \\ ${ }^{3}$ School of Mathematical Sciences, University College Dublin, Belfield, \\ Dublin 4, Ireland \\ E-mail: watts@thphys.nuim.ie and jiri.vala@nuim.ie
}

Received 11 May 2011

Accepted 6 June 2011

Published 30 June 2011

Online at stacks.iop.org/JSTAT/2011/P06020

doi:10.1088/1742-5468/2011/06/P06020

\begin{abstract}
We describe how the Kitaev honeycomb model with a finite number of sites on a torus may be easily generalised to cylindrical and rectangular topologies using a recently developed fermionisation technique. We then look for criteria which determine if zero-energy modes exist for these cases in the absence of an external magnetic field. For the cylindrical case, we determine the combination of spin-spin coupling strengths and vortex configurations which give zero modes and construct them explicitly, and for the rectangular case we show that no zero modes can exist.
\end{abstract}

Keywords: solvable lattice models 


\section{Contents}

1. Introduction 2

2. Review of the model 3

3. Toroidal and cylindrical topologies 4

4. Existence of zero-energy states 8

$\begin{array}{ll}\text { 5. Cylindrical case } & 10\end{array}$

5.1. Criteria for zero-energy states . . . . . . . . . . . . . . . 10

5.2. Discussion and examples . . . . . . . . . . . . . . . . . . . 11

$\begin{array}{ll}\text { 6. Rectangular case } & 13\end{array}$

7. Conclusions 13

$\begin{array}{ll}\text { Acknowledgments } & 14\end{array}$

$\begin{array}{ll}\text { Appendix } & 14\end{array}$

$\begin{array}{ll}\text { References } & 18\end{array}$

\section{Introduction}

Detailed understanding of the physical conditions which enable formation of zeroenergy modes presents both formidable challenges and interesting applications. For example, Majorana zero-energy modes have attracted considerable attention recently, as they exhibit non-Abelian fractional statistics [1] which offer attractive applications in topological quantum information processing $[2,3]$ by providing intrinsic protection of quantum information against noise. They are believed to be realised as edge states in fractional quantum Hall systems with filling $\nu=5 / 2$ and in p-wave superconductors.

Zero-energy modes, and particularly Majorana modes, have also been investigated in the more abstract setting of lattice models with topological phases, particularly in the context of the Kitaev honeycomb lattice model [4] subjected to a weak magnetic field, which exhibits a non-Abelian topological phase of the Ising type. Such modes are also found in the large-size (thermodynamic) limit [5], where they are localised on edges. The relative simplicity of the model allows for its possible realisation in atomic [6] and molecular systems [7]; the initial step in doing so will likely be a proof-of-principle realisation of the model for a small finite size. It appears conceivable that in that case the existence of zero modes may be verified using, for example, spectroscopy.

Here we present a purely analytical treatment of the Kitaev honeycomb lattice model and determine the criteria under which zero-energy modes can occur in the specific case where the lattice has a finite number of sites (and thus its spectrum is gapped) and there is no external magnetic field. We emphasise that these zero-energy modes are different from the Majorana modes of the Ising topological phase of the model, but we believe their existence still remains interesting: they are exact zero-energy modes and they can be 
fully characterised analytically in some situations. Specifically, we provide some definite analytical results for several topologies: toroidal, cylindrical (or, equivalently, annular) and rectangular. Moreover, the existence of these zero-energy modes is independent of certain bulk properties, which suggests that they may be localised on boundaries rather than in the interior of the lattice. They may also emerge in finite-size realisations of the Kitaev honeycomb model.

In section 2, we present a brief review of the model. The reader is referred to [4] for the original approach, and to $[8,5]$ and the references therein for the particular formulation and notation we use here. In section 3, we discuss the toroidal and cylindrical cases in the context of our formulation and show how results in the former can lead to results in the latter. Section 4 deals with the criteria for the existence of zero-energy modes in the cylindrical topology along with a technique for actually constructing them. In doing so, we find definite restrictions on the possible boundary conditions and vortex configurations based on the size of our cylinder. We finish with some comments on what happens when we have a rectangular lattice.

\section{Review of the model}

The honeycomb model proposed in [4] places the spins at the vertices of a hexagonal lattice. Each spin is coupled to its three nearest neighbours according to their relative locations, labelled the $x, y$ and $z$ directions, such that the lattice Hamiltonian is given by

$$
H_{0}=-\sum_{\alpha=x, y, z} \sum_{i, j} J_{\alpha} K_{i, j}^{\alpha}
$$

where $K_{i, j}^{\alpha}=\sigma_{i}^{\alpha} \sigma_{j}^{\alpha}$ gives the nearest-neighbour interaction between spins at adjacent sites $i$ and $j$. The model with Hamiltonian $H_{0}$ exhibits one gapless phase and three Abelian topological phases which are unitarily equivalent to the toric code on a square lattice [9] in the fourth order in perturbation theory. The addition of a weak magnetic field results in turning the gapless phase into a non-Abelian topological phase of the Ising universality class.

In [8], a novel exact solution of the model was presented that provides a unified description of both Abelian and non-Abelian topological phases and allows the detailed investigation of zero modes. The model is first exactly mapped onto a new representation given by effective spins and hardcore bosons on a square lattice [10] and then fermionised using a Jordan-Wigner type fermionisation procedure. The resulting fermionic Hamiltonian is

$$
H=\frac{1}{2} \sum_{\vec{q}, \vec{q}^{\prime}}\left(\begin{array}{cc}
c_{\vec{q}}^{\dagger} & c_{\vec{q}}
\end{array}\right)\left(\begin{array}{cc}
\xi_{\vec{q} \vec{q}^{\prime}} & \Delta_{\vec{q} \vec{q}^{\prime}} \\
\Delta_{\vec{q} \vec{q}^{\prime}}^{\dagger} & -\xi_{\vec{q} \vec{q}^{\prime}}^{T}
\end{array}\right)\left(\begin{array}{c}
c_{\vec{q}^{\prime}} \\
c_{\vec{q}^{\prime}}^{\dagger}
\end{array}\right),
$$

where $\vec{q}=\left(q_{x}, q_{y}\right)$ labels the $N=N_{x} N_{y}$ points on the lattice (assumed to be finite). The $N \times N$ matrices $\xi$ and $\Delta$ are, respectively, Hermitian and antisymmetric operator-valued matrices which depend on the strengths $J_{x, y, z}$ of the spin-spin couplings. (They also depend on the strength of any applied magnetic field; however, we only consider the case when there is no such field.) 
The eigenstates of the above Hamiltonian will depend on the fermion content at each point on the lattice, and also the vortex content of each plaquette and the boundary conditions of the states which are described below. The first of these is explicitly encoded in the operators $c_{\vec{q}}$ and $c_{\vec{q}}^{\dagger}$ which satisfy the familiar anticommutation relations

$$
\left\{c_{\vec{q}}, c_{\vec{q}^{\prime}}\right\}=\left\{c_{\vec{q}}^{\dagger}, c_{\vec{q}^{\prime}}^{\dagger}\right\}=0, \quad\left\{c_{\vec{q}}, c_{\vec{q}^{\prime}}^{\dagger}\right\}=\delta_{\vec{q}, \vec{q}^{\prime}}
$$

and thus respectively annihilate and create fermions at lattice site $\vec{q}$.

An important role in the investigation of the model is played by the vortex operators. If the vertices of a given hexagon are labelled in a clockwise direction starting with the bottommost one (at position $\vec{q}$ ), then the vortex operators are defined as

$$
W_{\vec{q}}=\sigma_{1}^{z} \sigma_{2}^{x} \sigma_{3}^{y} \sigma_{4}^{z} \sigma_{5}^{x} \sigma_{6}^{y} .
$$

These operators all commute with each other and square to unity. If there is a vortex on the plaquette, then $W_{\vec{q}}=-1$, and if there is not, $W_{\vec{q}}=+1$. They naturally extend into the fermionic representation where the operator $W_{\vec{q}}$ is associated with the square plaquette labelled by the point $\vec{q}$ at its lower left-hand corner.

The $W$ operators and their eigenvalues are not independent on a torus: their product must be equal to unity. Furthermore, if both $N_{x}$ and $N_{y}$ are even, then the torus may be bicoloured in a chequerboard fashion and the products of the 'black' plaquettes and the 'red' plaquettes must separately be equal to unity.

In addition to the vortex operators, the boundary conditions need to be considered in the case of nonplanar topologies like the torus or cylinder. These are defined through two operators $\ell_{0}^{(x)}$ and $\ell_{0}^{(y)}$ which tell us the boundary conditions in the $x$ and $y$ directions. These have eigenvalues \pm 1 which, after fermionisation, correspond respectively to antiperiodic or periodic boundary conditions on the wavefunction. For example, a fermionic wavefunction with $\ell_{0}^{(x)}=+1$ and $\ell_{0}^{(y)}=-1$ is antiperiodic in the $x$ direction and periodic in the $y$ direction.

Thus, the eigenstates of $H$ will be labelled by the eigenvalues of $\ell_{0}^{(x)}$ and $\ell_{0}^{(y)}$ (boundary conditions) as well as the value of each $W_{\vec{q}}$ (vortex configuration, subject to the aforementioned constraints). The vortices and boundary conditions appear in the fermionic Hamiltonian above through the matrices $\xi$ and $\Delta$; we shall explicitly state how these depend on vortex configurations and boundary conditions in section 3 .

\section{Toroidal and cylindrical topologies}

A considerable body of work using the Kitaev honeycomb model has been done for systems which are toroidal, namely where the states are periodic or antiperiodic in both the $x$ and $y$ directions. We now discuss how we incorporate these two periodicities in our particular case, and how, from this, we may also get a model which is (anti)periodic in only one of the directions, i.e. a cylindrical model.

The toroidal nature of the system is given by identifying $q_{x}+N_{x}$ with $q_{x}$ and $q_{y}+N_{y}$ with $q_{y}$. We adopt the convention that the fundamental region is $q_{x}=0,1, \ldots, N_{x}-1$ and $q_{y}=0,1, \ldots, N_{y}-1$. However, for our matrix indices, we choose the following ordering: the site at $\left(q_{x}, q_{y}\right)$ corresponds to the index $q_{x}+N_{x} q_{y}+1$. In other words, we start with site 1 at $(0,0)$, move across in the $x$ direction to site $N_{x}$ at $\left(N_{x}-1,0\right)$, then move back and up so that site $N_{x}+1$ is at $(0,1)$. We continue moving to the right and up until we reach the final site $N_{x} N_{y}$ at $\left(N_{x}-1, N_{y}-1\right)$ (see figure 1$)$. In this basis, we find that the 


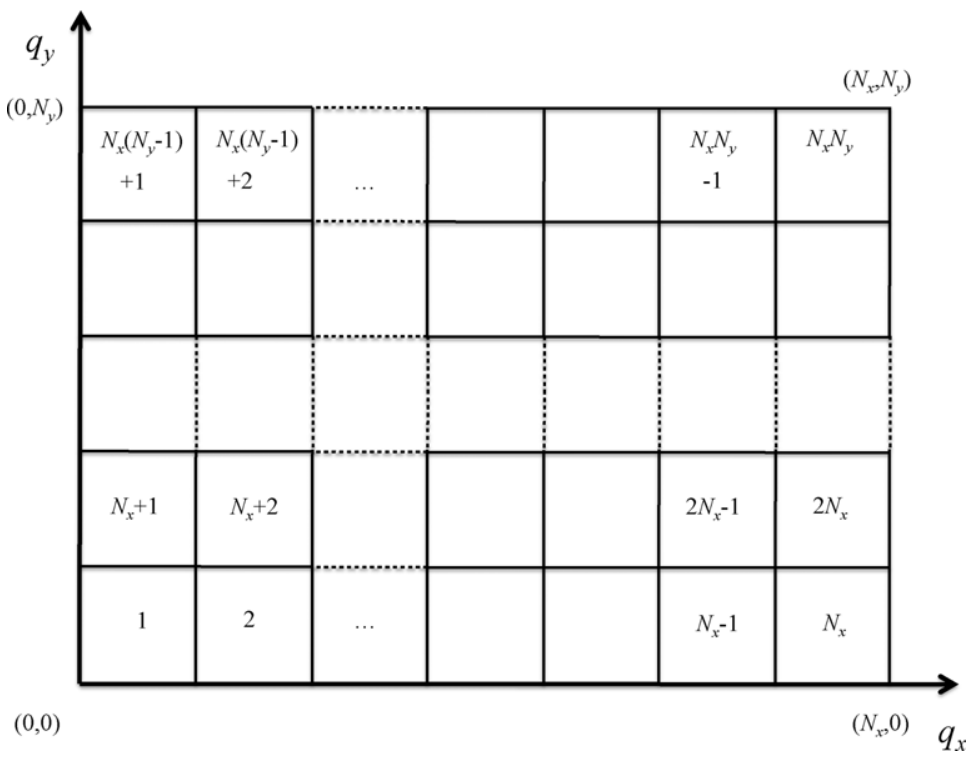

Figure 1. Plaquette ordering. The indices of the matrices $\xi$ and $\Delta$ in the Hamiltonian label the plaquettes as shown above. Note that, for the toroidal topology, the left and right sides are identified with one another, as are the top and bottom edges.

matrices $\xi$ and $\Delta$ in $(2.2)$ are

$$
\xi=\frac{1}{2}\left(A+A^{\mathrm{T}}\right), \quad \Delta=\frac{1}{2}\left(A-A^{\mathrm{T}}\right)
$$

where $A$ is the real matrix with entries $A_{i j}$ given by

$$
\begin{aligned}
& 2 J_{x} X_{i} \quad \text { when } i=r N_{x}, \quad j=(r-1) N_{x}+1 \text { for } r=1, \ldots, N_{y}, \\
& 2 J_{y} Y_{i} \quad \text { when } i=\left(N_{y}-1\right) N_{x}+r, \quad j=r \text { for } r=1, \ldots, N_{x}, \\
& 2 J_{z} \delta_{i j}+2 J_{x} X_{i} \delta_{i+1, j}+2 J_{y} Y_{i} \delta_{i+N_{x}, j} \quad \text { otherwise, }
\end{aligned}
$$

where $X_{i}$ and $Y_{i}$ are operators whose values depend on the plaquette and boundarycondition operators. When $i=1, \ldots, N_{x}-1, X_{i}=1$; when $i$ is a multiple of $N_{x}$ (i.e. along the right edge), then

$$
X_{r N_{x}}= \begin{cases}-\ell_{0}^{(x)} & r=1 \\ -\ell_{0}^{(x)} \prod_{q_{y}^{\prime}=0}^{r-2} W_{\left(N_{x}-1, q_{y}^{\prime}\right)} & r=2, \ldots, N_{y}\end{cases}
$$

and when $i$ is any other value, expressed as $q_{x}+N_{x} q_{y}+1$ with $q_{x} \neq N_{x}-1$ and $q_{y} \neq 0$, then

$$
X_{q_{x}+N_{x} q_{y}+1}=\prod_{q_{y}^{\prime}=0}^{q_{y}-1} W_{\left(q_{x}, q_{y}^{\prime}\right)} .
$$


The $Y$ operators are simpler: when $i$ is a site anywhere other than the top edge, $Y_{i}=1$. On the top edge, where $q_{y}=N_{y}-1, i$ has the form $N_{x}\left(N_{y}-1\right)+r$ for $r=1, \ldots, N_{x}$, and

$$
Y_{N_{x}\left(N_{y}-1\right)+r}=-\ell_{0}^{(y)} \prod_{q_{x}=0}^{r-2} \prod_{q_{y}=0}^{N_{y}-1} W_{\left(q_{x}, q_{y}\right)} .
$$

Another way of thinking about the matrix $A$ is to write it in terms of blocks: it is an $N \times N$ matrix which can be broken down into an $N_{y} \times N_{y}$ matrix whose entries are themselves $N_{x} \times N_{x}$ submatrices. Only $2 N_{y}$ of these submatrices are nonzero; the diagonal entries $\mathcal{A}_{1}, \ldots, \mathcal{A}_{N_{y}}$, the submatrices $\mathcal{D}_{1}, \ldots, \mathcal{D}_{N_{y}-1}$ immediately above the diagonal and the submatrix $\mathcal{D}_{N_{y}}$ in the lower left-hand corner of $A$ :

$$
A=\left(\begin{array}{ccccc}
\mathcal{A}_{1} & \mathcal{D}_{1} & 0 & \ldots & 0 \\
0 & \mathcal{A}_{2} & \mathcal{D}_{2} & \ldots & 0 \\
\cdot & \cdot & \cdot & \cdot & \cdot \\
0 & 0 & \ldots & \mathcal{A}_{N_{y}-1} & \mathcal{D}_{N_{y}-1} \\
\mathcal{D}_{N_{y}} & 0 & \ldots & 0 & \mathcal{A}_{N_{y}}
\end{array}\right)
$$

In the basis we have chosen, the form of the $N_{x} \times N_{x}$ submatrices are reasonably simple: the $\mathcal{D}$ s are purely diagonal, with entries

$$
\left(\mathcal{D}_{r}\right)_{a b}=2 J_{y} Y_{(r-1) N_{x}+a} \delta_{a b} .
$$

The $\mathcal{A}$ s have the same general form that $A$ itself does: the only nonzero entries of $\mathcal{A}_{r}$ are the diagonal elements $2 J_{z}$, the entries $2 J_{x} X_{(r-1) N_{x}+1}$ through $2 J_{x} X_{(r-1) N_{x}+N_{x}-1}$ immediately above the diagonal and $2 J_{x} X_{(r-1) N_{x}+N_{x}}$ in the lower left-hand corner:

$$
\left(\mathcal{A}_{r}\right)_{a b}= \begin{cases}2 J_{z} & \text { for } a=b, \quad a=1, \ldots N_{x} \\ 2 J_{x} X_{r+a-1} & \text { for } a=b-1, \quad a=1, \ldots N_{x}-1 \\ 2 J_{x} X_{r+N_{x}-1} & \text { for } a=N_{x}, \quad b=1 .\end{cases}
$$

When we are on a torus, all the $X$ and $Y$ operators will be \pm 1 , depending on the periodicities and vortex configuration we have. However, note that the operator $\ell_{0}^{(y)}$ appears only in those terms in the Hamiltonian connecting the sites at $\left(q_{x}, 0\right)$ to $\left(q_{x}, N_{y}-1\right)$. In our basis, these correspond to the $i=\left(N_{x}-1\right) N_{y}+q_{x}+1, j=q_{x}+1$ entries of $A$, which are precisely the entries in $\mathcal{D}_{N_{y}}$. Severing these links -i.e. setting the coupling strengths between them to zero - is thus completely equivalent to taking $\ell_{0}^{(y)}=0$ (figure 2). In other words, all results obtained for our Hamiltonian expressed in terms of $\ell_{0}^{(y)}$ are not only valid on a torus, with $\ell_{0}^{(y)}= \pm 1$ as appropriate, but also for a cylinder when $\ell_{0}^{(y)}=0$. Periodicity in the $x$ direction is still described by $\ell_{0}^{(x)}= \pm 1$, but now our system has a finite height $N_{y}-1$ in the $y$ direction. This is not saying that the original operator $\ell_{0}^{(y)}$ has zero as an eigenvalue; rather that it does not appear in the Hamiltonian at all when we have a cylinder. Taking $\ell_{0}^{(y)}=0$ is simply a convenient and consistent way of setting all coupling strengths between the top and bottom edges to zero. (Although these edges are straight when the plaquettes are represented as squares, they are in fact zigzag-shaped in the original hexagonal lattice.) 


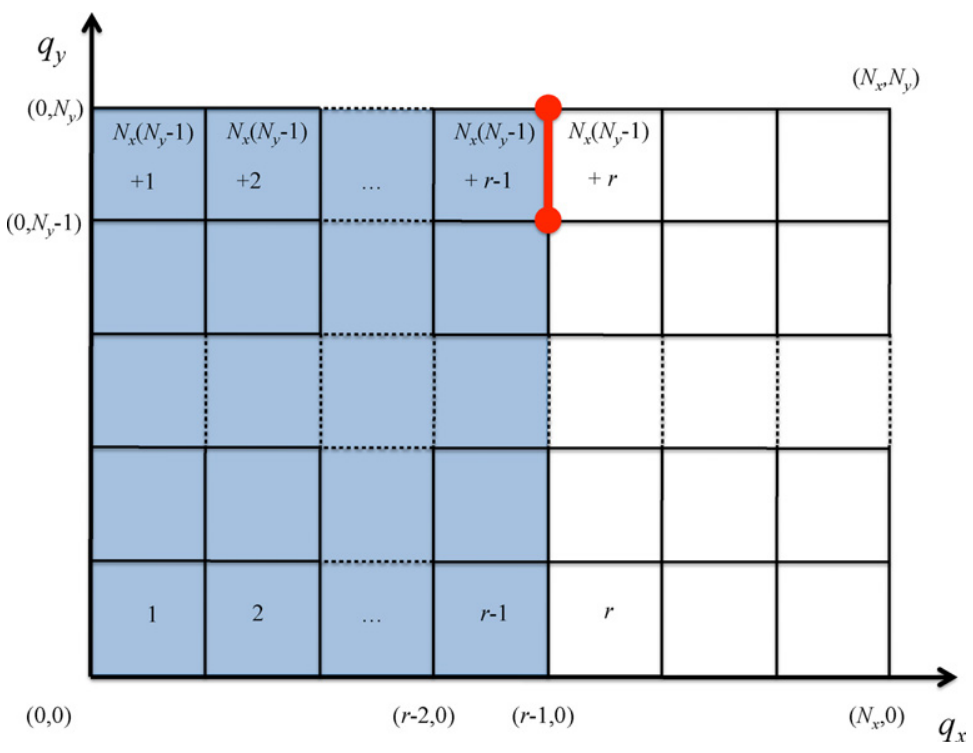

Figure 2. Coupling between edges. The highlighted (red) link above gives the coupling between the site at $\left(r-1, N_{y}-1\right)$ and the site at $\left(r-1, N_{y}\right)=(r-1,0)$. Its strength is $-J_{y} \ell_{0}^{(y)}$ times the values of the plaquette operators of the shaded region. Thus, severing this link is equivalent to setting $\ell_{0}^{(y)}$ to zero.

The usefulness of the basis we have chosen may now be seen by noting that, if $\ell_{0}^{(y)}=0$, then $\mathcal{D}_{N_{y}}$ also vanishes and $A$ is block upper-triangular. Manipulating such matrices is often easier than non-upper-triangular ones, and we shall see that this is indeed the case when we look at the criteria for zero-energy states.

One important point to remember, though: for a torus, we also had constraints on the plaquette operators $W_{\vec{q}}$, namely that their product was unity on a general torus, and, if the torus was bicolourable, that each of the 'black' and 'red' plaquette products was separately unity. However, on a cylinder, these constraints no longer apply: any vortex configuration is now possible. Thus, simply setting $\ell_{0}^{(y)}$ to zero for any toroidal result gives a valid result on a cylinder only for a particular vortex configuration which satisfies the plaquette constraints just mentioned. We by no means obtain any information for an arbitrary energy eigenstate of our cylindrical lattice.

We could, of course, have taken $\ell_{0}^{(x)}=0$ to obtain a cylinder circular in the $y$ direction and of width $N_{x}-1$ in the $x$ direction. Although $A$ looks somewhat different in this case it is not block upper-triangular, although the individual $\mathcal{A}$ submatrices are-all the results are precisely the same as for the $\ell_{0}^{(y)}=0$ case with the $x$ and $y$ directions swapped. This symmetry allows us to define, without loss of generality, a cylindrical topology as one with $\ell_{0}^{(y)}=0$ and $\ell_{0}^{(x)}= \pm 1$.

As a last comment in this section, note that we could of course set both $\ell_{0}^{(x)}$ and $\ell_{0}^{(y)}$ to zero, which results in a rectangular system of width $N_{x}-1$ and height $N_{y}-1$. Thus, we could obtain information about such states from a cylindrical lattice with $\ell_{0}^{(y)}=0$ by leaving $\ell_{0}^{(x)}$ arbitrary, and then setting $\ell_{0}^{(x)}$ to zero. In this case, we need not worry about relaxing any constraints on the vortex configuration. 


\section{Existence of zero-energy states}

Now that we have quantified how toroidal, cylindrical and rectangular topologies are incorporated into our model, we now determine the conditions under which we can find states which have zero energy. In this section, we look at the most general topology, the toroidal.

The key to finding zero-energy states for any of the topologies lies in the fact that the matrix appearing in (2.2) has eigenvalues which appear in $N$ pairs $E$ and $-E$; if we label the nonnegative members of each pair as $E_{n}$ for $n=1, \ldots, N$, then

$$
\begin{aligned}
\operatorname{det}_{2 N}\left(\begin{array}{cc}
\xi & \Delta \\
\Delta^{\dagger} & -\xi^{\mathrm{T}}
\end{array}\right) & =\prod_{n=1}^{N} E_{n} \prod_{n=1}^{N}\left(-E_{n}\right) \\
& =(-1)^{N}\left(\prod_{n=1}^{N} E_{n}\right)^{2} .
\end{aligned}
$$

The requirement for the existence of zero-energy states is therefore the vanishing of this determinant.

However, note that the above matrix is similar to one whose entries involve only the matrix $A$ :

$$
\left(\begin{array}{cc}
\xi & \Delta \\
\Delta^{\dagger} & -\xi^{\mathrm{T}}
\end{array}\right)=S \cdot \mathbb{A} \cdot S^{-1}
$$

where

$$
S:=\frac{1}{\sqrt{2}}\left(\begin{array}{cc}
I & I \\
-I & I
\end{array}\right)
$$

and

$$
\mathbb{A}:=\left(\begin{array}{cc}
0 & A \\
A^{\mathrm{T}} & 0
\end{array}\right)
$$

Thus,

$$
\operatorname{det}_{2 N} \mathbb{A}=(-1)^{N}\left(\prod_{n=1}^{N} E_{n}\right)^{2} .
$$

But the determinant of a purely block off-diagonal matrix is easily computed:

$$
\begin{aligned}
\operatorname{det}_{2 N}\left(\begin{array}{cc}
0 & A \\
A^{\mathrm{T}} & 0
\end{array}\right) & =\operatorname{det}_{N}\left(-A A^{\mathrm{T}}\right) \\
& =(-1)^{N}\left(\operatorname{det}_{N} A\right)^{2} .
\end{aligned}
$$

Therefore

$$
\prod_{n=0}^{N} E_{n}=\left|\operatorname{det}_{N} A\right| .
$$


We conclude that a zero-energy mode is only possible when the determinant of $A$ vanishes. This requires the existence of a nonzero vector $w$ such that $A \cdot w=0$; if we write $w$ as

$$
w=\left(\begin{array}{c}
w_{1} \\
w_{2} \\
\cdot \\
\cdot \\
\cdot \\
w_{N_{y}}
\end{array}\right)
$$

where each $w_{r}$ is an $N_{x}$-dimensional column vector, then the condition that $w$ is a null eigenvector of $A$ becomes

$$
\begin{array}{lc}
\mathcal{A}_{r} \cdot w_{r}+\mathcal{D}_{r} \cdot w_{r+1}=0 & \text { for } r=1, \ldots, N_{y}-1, \\
\mathcal{A}_{N_{y}} \cdot w_{N_{y}}+\mathcal{D}_{N_{y}} \cdot w_{1}=0 & \text { otherwise. }
\end{array}
$$

Since all of the $Y \mathrm{~s}$ appearing in $\mathcal{D}_{1}$ through $\mathcal{D}_{N_{y}-1}$ are nonzero, these matrices are invertible (in fact, $Y_{\vec{q}}=1$ in all these matrices, meaning that each one is simply $2 J_{y}$ times the $N_{x} \times N_{x}$ identity matrix); this means that we can eliminate all but $w_{1}$ from the above equations to obtain

$$
\left[\mathcal{D}_{N_{y}}-(-1)^{N_{y}} \mathcal{A}_{N_{y}} \cdot \mathcal{D}_{N_{y}-1}^{-1} \cdot \mathcal{A}_{N_{y}-1} \cdot \ldots \cdot \mathcal{D}_{1}^{-1} \cdot \mathcal{A}_{1}\right] \cdot w_{1}=0 .
$$

If $w_{1}=0,(4.11)$ implies that $w_{r}=0$ for all $r$, so if we require a nontrivial solution to $A \cdot w=0$, we see that the vanishing of the determinant of $A$ implies

$$
\operatorname{det}_{N_{x}}\left[\mathcal{D}_{N_{y}}-(-1)^{N_{y}} \mathcal{A}_{N_{y}} \cdot \mathcal{D}_{N_{y}-1}^{-1} \cdot \mathcal{A}_{N_{y}-1} \cdot \ldots \cdot \mathcal{D}_{1}^{-1} \cdot \mathcal{A}_{1}\right]=0 .
$$

This is therefore a necessary and sufficient condition that must be satisfied for the existence of zero-energy eigenstates. The determinant is an $N_{x}$ th-degree polynomial in $\ell_{0}^{(x)}$ and $\ell_{0}^{(y)}$, and its vanishing thus imposes constraints on the vortex configuration and couplingconstant strengths for toric $\left(\ell_{0}^{(x, y)}= \pm 1\right)$, cylindrical $\left(\ell_{0}^{(x)}= \pm 1, \ell_{0}^{(y)}=0\right)$ and rectangular $\left(\ell_{0}^{(x)}=\ell_{0}^{(y)}=0\right)$ topologies.

The toroidal case is unsurprisingly the most difficult, and it is not obvious that we can compute the above determinant analytically for systems of arbitrary size, vortex configuration and coupling strengths. For this topology, the only way to find the particular states for which the determinant vanishes may be through numerical methods. On the other hand, we can learn a great deal about the cylindrical and rectangular cases purely analytically, and so we concentrate on these cases in sections 5 and 6 .

Before doing so, it is worth emphasising that we consider only systems of finite size and with no external magnetic field. This makes our model fundamentally different from some others which predict zero-energy modes; for example, it has been shown [11] that, if there are an even number of vortices on a torus, there are an equal number of fermionic modes whose energies tend exponentially towards zero as the separation between vortices increases, but this occurs only for a nonzero magnetic field in the thermodynamic limit. Thus, any comparison between such results and any we would obtain using (4.13) to find zero-energy states on a torus would have to be done with a degree of caution, and so we do not attempt to make any such connections in this work. 


\section{Cylindrical case}

Equation (4.13) holds for all topologies, but unlike the toroidal case, where finding configurations which satisfy this condition analytically may be very difficult, we now show that we can find exact solutions if we go to a cylindrical topology where $\ell_{0}^{(y)}=0$. If we make this assumption, then $\mathcal{D}_{N_{y}}=0$ and, since all of the other $\mathcal{D}$ s have nonzero determinant, (4.13) implies

$$
\prod_{r=1}^{N_{y}} \operatorname{det}_{N_{x}} \mathcal{A}_{r}=0 .
$$

So at least one of the $\mathcal{A}$ submatrices must have zero determinant, and thus a null eigenvector. Determining the conditions under which this can occur involves some relatively straightforward but lengthy calculations, which are detailed in the appendix; here we just present the main results.

\subsection{Criteria for zero-energy states}

The coupling strengths in the $x$ and $z$ directions, $J_{x}$ and $J_{z}$, must have the same magnitude, with no restriction on $J_{y}$. (By convention, we assume the spin-spin interaction is ferromagnetic and for the rest of this discussion take $J_{x}=J_{z}=J>0$.) The fact that $J_{y}$ plays no role strongly suggests that any zero-energy states do not exist in the interior of the cylinder (where all the $y$ couplings are) but rather on the boundaries.

The vortex configurations which permit zero-energy states are characterised by two integers $R$ and $\bar{R}$, which take on values from 1 to $N_{y}$ subject to the constraint $R \leq \bar{R}$. These vortex configurations must be one of three types:

(i) $R=1$ : if $N_{x}$ is odd, then $\ell_{0}^{(x)}=+1$; if $N_{x}$ is even, $\ell_{0}^{(x)}=-1$. For either case, there must be an even number of vortices in total between the cylinder's lower edge and $q_{y}=\bar{R}-1$, an odd number in the strip between $\bar{R}-1$ and $\bar{R}$, and an even number in each individual strip from $\bar{R}$ to the cylinder's top edge.

(ii) $R=2, \ldots, N_{y}-1$ : if $N_{x}$ is odd, then $\ell_{0}^{(x)}=-1$; if $N_{x}$ is even, $\ell_{0}^{(x)}=+1$. For either case, there must be an even number of vortices in each strip from the cylinder's lower edge to $q_{y}=R-2$, an odd number in the strip between $R-2$ and $R-1$, an even number in total between $R-1$ and $\bar{R}-1$, an odd number between $\bar{R}-1$ and $\bar{R}$, and an even number in each individual strip from $\bar{R}$ to the cylinder's top edge.

(iii) $R=\bar{R}=N_{y}$ : if $N_{x}$ is odd, then $\ell_{0}^{(x)}=-1$; if $N_{x}$ is even, $\ell_{0}^{(x)}=+1$. For either case, there must be an even number of vortices in each strip from the cylinder's lower edge to $q_{y}=N_{y}-2$ and an odd number in the strip between $N_{y}-2$ and the cylinder's top edge at $q_{y}=R-1$.

For a given $(R, \bar{R})$ vortex configuration, the null eigenvectors $\omega^{(R, \bar{R})}$ of $\mathbb{A}$ are

$$
\omega^{(R, \bar{R})}=\left(\begin{array}{l}
\bar{w}^{(\bar{R})} \\
w^{(R)}
\end{array}\right) .
$$


where

$$
\bar{w}^{(\bar{R})}=\left(\begin{array}{c}
0 \\
\cdot \\
0 \\
\bar{w}_{\bar{R}}^{*} \\
\bar{w}_{\bar{R}+1}^{(\bar{R})} \\
\bar{w}_{N_{y}}^{(\bar{R})}
\end{array}\right), \quad w^{(R)}=\left(\begin{array}{c}
w_{1}^{(R)} \\
w_{2}^{(R)} \\
\cdot \\
w_{R}^{*} \\
0 \\
\cdot \\
0
\end{array}\right) .
$$

The components of these vectors are determined by the vortex configuration via

$$
w_{R}^{*} \propto\left(\begin{array}{c}
1 \\
-X_{R} \\
X_{R} X_{R+1} \\
\cdot \\
\cdot \\
\cdot \\
-(-1)^{N_{x}} X_{R} X_{R+1} \ldots X_{R+N_{x}-2}
\end{array}\right),
$$

$\bar{w}_{\bar{R}}^{*}$ is found by replacing $R$ by $\bar{R}$ in the above, and the other components are given by

$$
\begin{aligned}
& w_{r}^{(R)}:=(-1)^{r-R-1} \mathcal{A}_{r}^{-1} \cdot \mathcal{D}_{r} \cdot \ldots \cdot \mathcal{A}_{R-1}^{-1} \cdot \mathcal{D}_{R-1} \cdot w_{R}^{*}, \\
& \bar{w}_{r}^{(\bar{R})}:=(-1)^{\bar{R}-r}\left(\mathcal{A}_{r}^{\mathrm{T}}\right)^{-1} \cdot \mathcal{D}_{r} \cdots\left(\mathcal{A}_{\bar{R}+1}^{\mathrm{T}}\right)^{-1} \cdot \mathcal{D}_{\bar{R}+1} \cdot \bar{w}_{\bar{R}}^{*} .
\end{aligned}
$$

\subsection{Discussion and examples}

We may use the criteria just presented to determine under what circumstances - if any - a given vortex configuration admits a zero-energy mode. For example, consider a cylinder with no vortices: if we already assume $J_{x}=J_{z}$, then a zero mode can exist if $R=1$ and $\bar{R}=N_{y}$, as this is the only case that allows an even number of vortices in the region between $q_{y}=0$ and $q_{y}=\bar{R}-1=N_{y}-1$, namely the entire cylinder. Then if $\left(N_{x}, \ell_{0}^{(x)}\right)$ is either (odd, +1 ) or (even, -1 ), a zero-energy mode exists. All $W$ operators are +1 in this case, and so all the $X$ operators are known and the $w$ and $\bar{w}$ vectors may be determined.

The existence of zero-energy modes for the case where every plaquette contains a vortex depends on $N_{x}$ : if it is even, then each strip has an even number of vortices in it. The exact same argument as that just presented for the zero-vortex case leads us to conclude that we will have a zero-energy state if $R=1, \bar{R}=N_{y}$ and $\ell_{0}^{(x)}=-1$.

If $N_{x}$ is odd, then each strip contains an odd number of vortices; this immediately implies that we may only have zero-energy modes for $\bar{R}=N_{y}$, because all other values require at least one strip with an even number of vortices between $\bar{R}-1$ and $N_{y}-1$. Similarly, sectors with $R>2$ are excluded, because these sectors require at least one strip with an even number of vortices between $q_{y}=0$ and $q_{y}=R-2$. We know that the region between $q_{y}=R-1$ and $q_{y}=N_{y}-2$ must contain an even number of vortices, and since this region contains $\left(N_{y}-R-1\right) N_{x}$ plaquettes in total, we conclude that $R=1$ if $N_{y}$ is even and $R=2$ if $N_{y}$ is odd. The former requires that $\ell_{0}^{(x)}$ is +1 if a zero-energy state is to exist, the latter that it is -1 . 


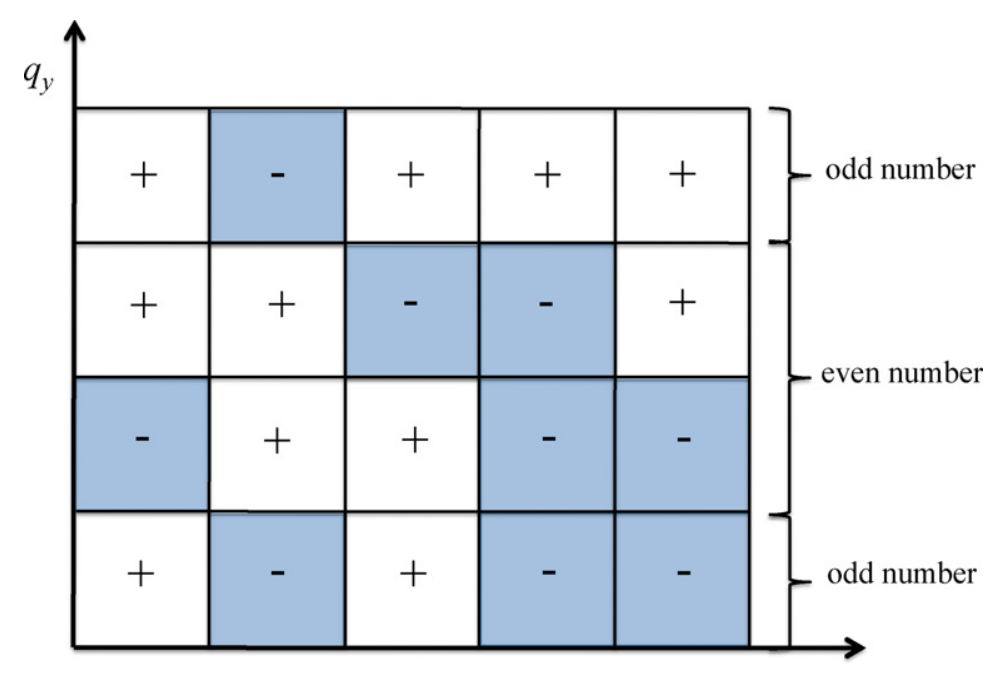

$q_{x}$

Figure 3. Zero-energy vortex configuration. The above figure wraps around horizontally but not vertically, and so describes an $N_{x}=N_{y}=5$ cylinder. The presence of a vortex on a plaquette is indicated by a minus sign, so if $\ell_{0}^{(x)}=-1$, then the above vortex configuration corresponds to an $R=2, \bar{R}=4$ zero-energy state.

An example of a vortex configuration - neither empty nor full-which admits a zeroenergy mode on an $N_{x}=5, N_{y}=5$ cylinder is given in figure 3 .

We may now find explicitly the creation and annihilation operators for the zero modes in any given $(R, \bar{R})$ sector: following the standard Bogoliubov-Hartree-Fock procedure (cf [12]), the Hamiltonian (2.2) becomes

$$
H=\sum_{i} E_{i}\left(a_{i}^{\dagger} a_{i}-\frac{1}{2}\right)
$$

where $a_{i}^{\dagger}$ and $a_{i}$ are the creation and annihilation operators of the fermionic eigenstates with (nonzero) energy $E_{i}$. The zero-mode operators are constructed as follows: the Hamiltonian $H$ is written in our basis as

$$
H=\frac{1}{4}\left(\begin{array}{cc}
c^{\dagger}-c & c^{\dagger}+c
\end{array}\right)\left(\begin{array}{cc}
0 & A \\
A^{\mathrm{T}} & 0
\end{array}\right)\left(\begin{array}{c}
c-c^{\dagger} \\
c+c^{\dagger}
\end{array}\right)
$$

(where the sum over lattice sites has been dropped for clarity). The matrix is diagonalised to the form $U \cdot \mathcal{E} \cdot U^{-1}$, where $\mathcal{E}$ is the diagonal matrix whose elements are the energy eigenvalues; therefore, if we choose a basis so that the zero eigenvalue appears as the first diagonal element in $\mathcal{E}$, then the first column of $U$ is the null eigenvector $\omega^{(R, \bar{R})}$. Therefore, the operators $\gamma$ and $\gamma^{\dagger}$ corresponding to the $(R, \bar{R})$ zero mode are given by

$$
\begin{aligned}
\gamma^{\dagger} & =\frac{1}{\sqrt{2}}\left(\begin{array}{ll}
c^{\dagger}-c & c^{\dagger}+c
\end{array}\right) \cdot\left(\begin{array}{c}
\bar{w} \\
w
\end{array}\right) \\
& =\frac{1}{\sqrt{2}}(\bar{w}+w) \cdot c^{\dagger}+\frac{1}{\sqrt{2}}(\bar{w}-w) \cdot c,
\end{aligned}
$$




$$
\gamma=\frac{1}{\sqrt{2}}(\bar{w}-w) \cdot c^{\dagger}+\frac{1}{\sqrt{2}}(\bar{w}+w) \cdot c .
$$

(We have suppressed the superscripts on $w^{(R)}$ and $\bar{w}^{(\bar{R})}$ for the sake of convenience.) It is straightforward to use (2.3) to show that

$$
\begin{aligned}
& \{\gamma, \gamma\}=\left\{\gamma^{\dagger}, \gamma^{\dagger}\right\}=|\bar{w}|^{2}-|w|^{2}, \\
& \left\{\gamma, \gamma^{\dagger}\right\} \quad=|\bar{w}|^{2}+|w|^{2} .
\end{aligned}
$$

Recall that $w$ and $\bar{w}$ were determined only up to a multiplicative factor, so if we normalise them so that they both have norm $1 / \sqrt{2}$ (and hence $|\omega|=1$ ), then we see that $\{\gamma, \gamma\}=\left\{\gamma^{\dagger}, \gamma^{\dagger}\right\}=0$ and $\left\{\gamma, \gamma^{\dagger}\right\}=1$, and these therefore may be interpreted as the zero-mode creation and annihilation operators for the $(R, \bar{R})$ vortex configuration.

\section{Rectangular case}

The remaining topology which is incorporated in our model is the rectangular one, in which we do not assume that states have any sort of periodic boundary conditions.

We have argued that, if $\ell_{0}^{(x)}$ is left as a variable in any calculations for a cylindrical topology, we can obtain results for a rectangle of size $\left(N_{x}-1\right) \times\left(N_{y}-1\right)$ simply by setting it to zero. Since we have left $\ell_{0}^{(x)}$ as unspecified in (A.4), we can conclude that the rectangular case permits no zero modes: setting $\ell_{0}^{(x)}=0$ immediately shows that each $\mathcal{A}_{r}$ has determinant $(2 J)^{N}$, and so the product of their determinants, which is also the product of the nonnegative half of the energy eigenvalues, is manifestly positive and so $E_{n}>0$ for all $n$. There can therefore be no zero-energy modes on a rectangular lattice in the absence of an external magnetic field.

\section{Conclusions}

We have explained how one can use the fermionisation scheme presented in [8] to obtain results about the finite Kitaev honeycomb model on cylindrical and rectangular lattices from a toroidal lattice. This is done by using the fact that setting one of the boundarycondition operators to zero is entirely equivalent to removing the links between a row of sites, and thus introducing an edge. This technique is general enough to include all vortex configurations and coupling strengths.

As the existence of zero-energy fermions in the model can be of great interest, we then set out to find the necessary criteria under which such states can exist for a lattice of finite size. Our choice of basis then allowed us to write down an equation which guarantees at least one vanishing energy eigenstate.

Although this equation arose from considering a toroidal system, it also applies to a cylindrical lattice in the manner described, and we showed that any existing zero modes were completely characterised by two integers (whose values depended on the size of the cylinder). We then explicitly constructed the operators which create and annihilate any one of these modes. We finished with a proof that there can be no zero-energy modes when the lattice is rectangular.

Although our results were completely analytical, they were necessarily limited; for example, although we made some brief comments about what happens when the topology 
is toroidal, we did not consider this case due to the difficulty of obtaining analytical results. Although we found the necessary criteria for the existence of a zero-energy state, actually applying it to tori with arbitrary boundary conditions, coupling strengths and vortex configurations seemed mathematically intractable. In principle, it should not be difficult to use numerical methods to obtain useful results, but that is beyond the scope of this paper.

However, even though we were far more successful in finding the exact conditions for which zero modes exist for cylindrical systems, it must be stressed that said conditions are necessary but possibly not restrictive enough. For instance, although we showed that there must be two integers $R$ and $\bar{R}$ associated with any zero modes such that $R \leq \bar{R}$, it may be that there are stronger restrictions on these two numbers due to other aspects of the model that we have not considered here.

Also, as the degeneracy of a given energy eigenstate is quite important in any theory (and can sometimes give very deep insight into the fundamental physics), counting up the different number of ways in which any of these zero-energy modes can be realised must be addressed. But, as with the necessary-versus-sufficient question just mentioned, our approach may not be fully considering all the facets of the model to allow us to assign a definite degeneracy to any one of our zero modes.

Throughout this work, we have assumed that there is no external magnetic field. The question of what happens to the zero modes we have found when such a field is present is a possible avenue for investigation. Because the terms introduced to the Hamiltonian by a weak magnetic field are similar to those already present, we feel that the approach described here may yield some exact results and hope to investigate this case in future.

Finally, merely showing the existence of zero-energy states still leaves open the question of what type of states they are. Even though we find these exist on a cylinder only when two of the coupling strengths have the same magnitude, the remaining coupling constant plays no role whatsoever, which strongly suggests that our states do not exist in the bulk of the lattice, but only on the cylinder's edges. Although this does not necessarily preclude zero modes localised around individual vortices, it seems more likely that they propagate purely along the circular boundaries of the cylinder. However, our analysis in this paper at no point makes a distinction between localised and nonlocalised zero-energy states, and so we cannot at present say anything more definite. Future research along the lines presented in this work is needed to address these issues.

\section{Acknowledgments}

This work has been supported by Science Foundation Ireland through the President of Ireland Young Researcher Award 05/YI2/I680 and the Principal Investigator Award 10/IN.1/I3013. The authors would like to thank Graham Kells and Joost Slingerland for their helpful comments.

\section{Appendix}

In this appendix, we present the details of the computations that lead us to the results summarised in section 5.1. 
The condition for the existence of zero-energy modes for a cylindrical topology is

$$
\prod_{r=1}^{N_{y}} \operatorname{det}_{N_{x}} \mathcal{A}_{r}=0
$$

and thus at least one of the $\mathcal{A}$ s must have zero determinant. The determinant of any of these submatrices is straightforward to compute from (3.8):

$$
\operatorname{det}_{N_{x}} \mathcal{A}_{r}=\left(2 J_{z}\right)^{N_{x}}-(-1)^{N_{x}}\left(2 J_{x}\right)^{N_{x}} \prod_{a=1}^{N_{x}} X_{r+a-1} .
$$

We can now see immediately one of the necessary conditions for the existence of zeroenergy modes: since all of the $X$ operators can only have values \pm 1 , this can never vanish unless $\left|J_{x}\right|=\left|J_{z}\right|$. We assume from now on that $J_{x}=J_{z}$.

The product of the $X$ operators is also straightforward to compute from (3.4): we first define

$$
\bar{X}_{r}:= \begin{cases}1 & \text { for } r=1, \\ \prod_{q_{y}=0}^{r-2} \prod_{q_{x}=0}^{N_{x}-1} W_{\vec{q}} & \text { for } r=2, \ldots, N_{y} .\end{cases}
$$

Pictorially, $\bar{X}_{r}$ is the product of all plaquette operators enclosed within the cylindrical strip between $q_{y}=0$ and $q_{y}=r-1$. With this

$$
\operatorname{det}_{N_{x}} \mathcal{A}_{r}=(2 J)^{N_{x}}\left[1+(-1)^{N_{x}} \ell_{0}^{(x)} \bar{X}_{r}\right] .
$$

Thus, $\mathcal{A}_{r}$ has zero determinant only when the lattice size, boundary conditions and vortex configuration are such that $(-1)^{N_{x}} \ell_{0}^{(x)} \bar{X}_{r}=-1$ (with the only other possible value, $2(2 J)^{N_{x}}$, occurring when $\left.(-1)^{N_{x}} \ell_{0}^{(x)} \bar{X}_{r}=+1\right)$. This implies the following: the characteristic equation for $\mathcal{A}_{r}$ is easily shown to be

$$
\operatorname{det}_{N_{x}}\left(\mathcal{A}_{r}-\lambda I\right)=(2 J)^{N_{x}}\left[\left(1-\frac{\lambda}{2 J}\right)^{N_{x}}+(-1)^{N_{x}} \ell_{0}^{(x)} \bar{X}_{r}\right] .
$$

When $(-1)^{N_{x}} \ell_{0}^{(x)} \bar{X}_{r}=-1, \lambda=0$ is a root of multiplicity one, so $\mathcal{A}_{r}$ has at most one null eigenvalue. Thus, there is only one null eigenvector (up to scalar multiplication) $w_{r}^{*}$ for each $\mathcal{A}_{r}$ when its determinant vanishes. In fact, this eigenvector is easily shown to be

$$
w_{r}^{*} \propto\left(\begin{array}{c}
1 \\
-X_{r} \\
X_{r} X_{r+1} \\
\cdot \\
\cdot \\
\cdot \\
-(-1)^{N_{x}} X_{r} X_{r+1} \ldots X_{r+N_{x}-2}
\end{array}\right)
$$

(where we have used $X_{i}^{2}=1$ and the zero-determinant condition $X_{r} \ldots X_{r+N_{x}-1}=$ $\left.-\ell_{0}^{(x)} \bar{X}_{r}=(-1)^{N_{x}}\right)$. 
We are now able to construct the null eigenvectors of $A$ explicitly: assume $R$ is the largest value of $r$ for which the subvectors $w_{r}$ do not vanish, i.e. $w_{R} \neq 0$ and $w_{r>R}=0$. (4.11) immediately implies that $\mathcal{A}_{r} \cdot w_{r}+\mathcal{D}_{r} \cdot w_{r+1}=0$ for $r=1, \ldots, R-1$ and $\mathcal{A}_{R} \cdot w_{R}=0$. By assumption, $w_{R}$ is nonzero, so this implies that $\mathcal{A}_{R}$ has determinant zero and $w_{R}$ is the null eigenvector $w_{R}^{*}$ of $\mathcal{A}_{R}$ given by (A.6). However, (4.11) may be used to show that

$$
w_{r}=(-1)^{r-s+1} \mathcal{D}_{r-1}^{-1} \cdot \mathcal{A}_{r-1} \cdot \ldots \cdot \mathcal{D}_{s}^{-1} \cdot \mathcal{A}_{s} \cdot w_{s}
$$

for all $r>s$, which implies that

$$
(-1)^{R-s+1} \mathcal{D}_{R-1}^{-1} \cdot \mathcal{A}_{R-1} \cdots \mathcal{D}_{s}^{-1} \cdot \mathcal{A}_{s} \cdot w_{s}=w_{R}^{*}
$$

for $s=1, \ldots, R-1$. Since $w_{R}^{*} \neq 0$, then the above implies not only that none of the subvectors $w_{1}$ through $w_{R-1}$ can be zero, but also that none of the other $\mathcal{A}$ submatrices can have vanishing determinant.

An important caveat: strictly speaking, the preceding statement is not true, as the matrix equation $M \cdot x=b$ can have solutions for $x$ even if $\operatorname{det} M=0$ provided that $b$ is a null eigenvector of the adjugate of $M$. However, since the eigenvalues of the plaquette operators are entirely independent in a cylindrical topology, the likelihood that $w_{N_{y}}$ is simultaneously a null eigenvector of $\mathcal{A}_{N_{y}}$ and the adjugate of $\mathcal{D}_{N_{y}-1}^{-1} \cdot \mathcal{A}_{N_{y}-1} \cdots \cdots \mathcal{D}_{s}^{-1} \cdot \mathcal{A}_{s}$ is small. This case - if it is even possible - may indeed be very interesting in its own right, but we do not consider it in this work, and we assume that (A.8) implies that all the matrices $\mathcal{A}_{s}$ for $s<R$ are invertible.

Since $w_{R}^{*}$ is known and all of the matrices on the left-hand side of (A.8) are assumed to be invertible, $w_{1}$ through $w_{R-1}$ are entirely determined. Furthermore, we know that each $\mathcal{A}_{r}$ has only one null eigenvector, so all null eigenvectors of $A$ are uniquely given by

$$
w^{(R)}=\left(\begin{array}{c}
w_{1}^{(R)} \\
w_{2}^{(R)} \\
\cdot \\
w_{R}^{*} \\
0 \\
\cdot \\
0
\end{array}\right),
$$

where $\mathcal{A}_{R}$ has zero determinant and null eigenvector $w_{R}^{*}$ given by (A.6), $\operatorname{det}_{N_{x}} \mathcal{A}_{r}=$ $2(2 J)^{N_{x}}$ for $r=1, \ldots, R-1$ and

$$
w_{r}^{(R)}:=(-1)^{r-R-1} \mathcal{A}_{r}^{-1} \cdot \mathcal{D}_{r} \cdots \mathcal{A}_{R-1}^{-1} \cdot \mathcal{D}_{R-1} \cdot w_{R}^{*} .
$$

Since $R=1, \ldots, N_{y}$, it follows that there are $N_{y}$ distinct null eigenvectors of $A$.

We have just determined the conditions for which $A$ has a null eigenvector; however, this does not fully specify the zero modes of the full Hamiltonian, since we need to find the conditions under which there exists a $2 N$-dimensional vector $\omega$ satisfying

$$
\mathbb{A} \cdot \omega=0 .
$$

If we denote the top $N$ components of $\omega$ by the vector $\bar{w}$ and the bottom $N$ by $w$, then the form of $\mathbb{A}$ from (4.5) gives the two equations

$$
A \cdot w=0, \quad A^{\mathrm{T}} \cdot \bar{w}=0 .
$$


We have already considered the first of these; we consider the second similarly, by first defining the $N_{x}$-dimensional vectors $\bar{w}_{r}$ with $r=1, \ldots, N_{y}$, by

$$
\bar{w}=\left(\begin{array}{c}
\bar{w}_{1} \\
\bar{w}_{2} \\
\cdot \\
\cdot \\
\cdot \\
\bar{w}_{N_{y}}
\end{array}\right)
$$

so that, when $\mathcal{D}_{N_{y}}=0$,

$$
\begin{aligned}
& \mathcal{A}_{1}^{\mathrm{T}} \cdot \bar{w}_{1}=0, \\
& \mathcal{A}_{r}^{\mathrm{T}} \cdot \bar{w}_{r}+\mathcal{D}_{r} \cdot \bar{w}_{r-1}=0
\end{aligned}
$$

for $r=2, \ldots, N_{y}$. (We have used the fact that each $\mathcal{D}$ is diagonal and thus is symmetric.) Now, by letting $\bar{R}$ be the smallest value of $r$ for which $\bar{w}_{r} \neq 0$, we can repeat the previous argument almost verbatim to find that $\mathcal{A}_{\bar{R}}^{\mathrm{T}}$ must be singular, $\bar{w}_{\bar{R}}$ must be its null eigenvector $\bar{w}_{\bar{R}}^{*}$ given by

$$
\bar{w}_{\bar{R}}^{*} \propto\left(\begin{array}{c}
1 \\
-X_{\bar{R}} \\
X_{\bar{R}} X_{\bar{R}+1} \\
\cdot \\
\cdot \\
\cdot \\
-(-1)^{N_{x}} X_{\bar{R}} \ldots X_{\bar{R}+N_{x}-2},
\end{array}\right)
$$

and all of the matrices $\mathcal{A}_{\bar{R}+1}^{\mathrm{T}}$ through $\mathcal{A}_{N_{y}}^{\mathrm{T}}$ must have nonvanishing determinant, giving the nonzero subvectors $\bar{w}_{r}^{(\bar{R})}$ as

$$
\bar{w}_{r}^{(\bar{R})}:=(-1)^{\bar{R}-r}\left(\mathcal{A}_{r}^{\mathrm{T}}\right)^{-1} \cdot \mathcal{D}_{r} \cdots\left(\mathcal{A}_{\bar{R}+1}^{\mathrm{T}}\right)^{-1} \cdot \mathcal{D}_{\bar{R}+1} \cdot \bar{w}_{\bar{R}}^{*}
$$

for $r=\bar{R}+1, \ldots, N_{y}$. These give the nonzero components of the null eigenvector $\bar{w}^{(\bar{R})}$ of $A^{\mathrm{T}}$.

Recall that the determinant of the transpose of a matrix is the same as the nontransposed one, so the preceding argument tells us that there has to be an $\bar{R}$ such that $\mathcal{A}_{\bar{R}}$ is singular but that $\mathcal{A}_{r}$ for $r=\bar{R}+1, \ldots, N_{y}$ are not. However, we had already determined that there must be an $R$ such that $\operatorname{det}_{N_{x}} \mathcal{A}_{R}=0$ but $\operatorname{det}_{N_{x}} \mathcal{A}_{r} \neq 0$ for $r=1, \ldots, R$. The only way to reconcile these two results is to require $R \leq \bar{R}$. Therefore, every zero mode is a linear combination of zero modes labelled by $R$ and $\bar{R}$ :

$$
\omega^{(R, \bar{R})}=\left(\begin{array}{c}
\bar{w}^{(\bar{R})} \\
w^{(R)}
\end{array}\right) .
$$

We can now use (A.4) and the above analysis to look at what combinations of cylinder size, boundary conditions and vortex configurations allow zero modes in our system. First, consider the $R=1$ zero mode: (A.4) tells us that $\operatorname{det}_{N_{x}} \mathcal{A}_{1}=0$ requires $(-1)^{N_{x}} \ell_{0}^{(x)} \bar{X}_{1}=-1$. But $\bar{X}_{1}=+1$, so we see that this mode is possible only if (i) $N_{x}$ is odd and our state is antiperiodic or (ii) $N_{x}$ is even and our state is periodic. Both of 
these are possible independent of the vortex configuration, so we need look at the values of $\bar{R} . \quad \bar{R}$ can take on any value for this case, and since we know that $(-1)^{N_{x}} \ell_{0}^{(x)}=-1$, then the vanishing of $\mathcal{A}_{\bar{R}}$ requires $\bar{X}_{\bar{R}}=+1$ and the nonvanishing of $\mathcal{A}_{r}$ for $r>\bar{R}$ means that $\bar{X}_{r}=-1$ for $r=\bar{R}+1, \ldots, N_{y}$. The first of these requires there be an even number of vortices in the region between the cylinder's lower edge and $q_{y}=\bar{R}-1$.

But recall that the $\bar{X}$ operators are built up from the bottom edge (at $q_{y}=0$ ) by one-plaquette-thick strips, so that $\bar{X}_{r}$ is the product of all the plaquette operators in the bottommost $r-1$ strips. Thus, the difference in the eigenvalues of $\bar{X}_{r}$ and $\bar{X}_{r+1}$ is the product of the plaquette operators in the strip between $q_{y}=r-1$ and $q_{y}=r$. Since $\bar{X}_{\bar{R}}$ is positive but $\bar{X}_{\bar{R}+1}$ is negative, this tells us that there must be an odd number of vortices in the strip between $\bar{R}-1$ and $\bar{R}$. However, since $\bar{X}_{r}$ does not change sign as $r$ increases from $\bar{R}+1$, this means that each additional strip must contain an even number of vortices.

When $R=2, \ldots, N_{y}-1,\left(\right.$ A.4) tells us that $\bar{X}_{R}=-(-1)^{N_{x}} \ell_{0}^{(x)}$ and $\bar{X}_{r}=(-1)^{N_{x}} \ell_{0}^{(x)}$ for $r=1, \ldots, R-1$. Putting $r=1$ into the second of these gives the opposite state as just described, i.e. either $N_{x}$-odd/periodic or $N_{x}$-even/antiperiodic. For either case, $(-1)^{N_{x}} \ell_{0}^{(x)}=+1$, which immediately tells us that $\bar{X}_{r}=+1$ for all $r$ from 1 to $R-1$, and $\bar{X}_{R}=-1$. Thus, each individual one-plaquette-thick strip from the bottom to $R-2$ must contain an even number of vortices while the one between $R-2$ and $R-1$ has an odd number.

The condition $(-1)^{N_{x}} \ell_{0}^{(x)}=+1$ also tells us that, for $\bar{R} \geq R, \bar{X}_{\bar{R}}=-1$ and $\bar{X}_{r}=+1$ for $r=\bar{R}+1, \ldots, N_{y}$. Since we already know that the region between the bottom and $R-1$ has an odd number of vortices, the region between $R-1$ and $\bar{R}-1$ contains an even number because $\bar{X}_{\bar{R}}$ is also negative. But $\bar{X}_{r}$ then becomes positive, so $\bar{R}-1$ to $\bar{R}$ has an odd number of vortices, and each individual strip thereafter has an even number.

The remaining case is $R=N_{y}$, which also requires $\bar{R}=N_{y}$. Thus, $(-1)^{N_{x}} \ell_{0}^{(x)}=+1$, $\bar{X}_{r}=+1$ for $r=1, \ldots, N_{y}-1$ and $\bar{X}_{R}=-1$. This requires every single strip between $q_{y}=0$ and $q_{y}=N_{y}-2$ to have an even number of vortices, but the topmost strip contains an odd number.

\section{References}

[1] Read N and Green D, 2000 Phys. Rev. B 6110267

[2] Freedman M H, Kitaev A, Larsen M J and Wang Z, 2003 Bull. Am. Math. Soc. 4031

[3] Nayak C, Simon S H, Stern A, Freedman M H and Das Sarma S, 2008 Rev. Mod. Phys. 801083

[4] Kitaev A, 2006 Ann. Phys., NY 3212

[5] Kells G and Vala J, 2010 Phys. Rev. B 82125122

[6] Duan L-M, Demler E and Lukin M D, 2003 Phys. Rev. Lett. 91090402

[7] Micheli A, Brennen G K and Zoller P, 2006 Nature Phys. 2341

[8] Kells G, Slingerland J K and Vala J, 2009 Phys. Rev. B 80125415

[9] Kitaev A, 2003 Ann. Phys., NY 3032

[10] Schmidt K P, Dusuel S and Vidal J, 2008 Phys. Rev. Lett. 100057208

[11] Volovik G E, 1993 JETP Lett. 57244

[12] Ring P and Schuck P, 2004 The Nuclear Many-Body Problem 3rd edn (Berlin: Springer) p 611 